\title{
Efectos de la mediación parental, habilidades digitales, género y condición socioeconómica en el uso de internet en niñas, niños y adolescentes $^{1}$
}

\author{
Effects of Parental Mediation, Digital Skills, Gender and Socioeconomic Status on the \\ Internet Uses of Children and Adolescents ${ }^{2}$
}

\section{Efeitos da mediação parental, habilidades digitais, gênero e condição socioeconômica no uso da Internet em crianças e adolescentes ${ }^{3}$}

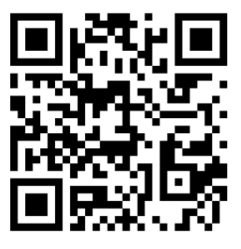

Corregido • Revised • Revisado: 14 / 07 / 2021

Aceptado • Accepted • Aprovado: 12 / 08 / 2021

\footnotetext{
${ }^{1}$ Este articulo es producto de un trabajo de colaboración entre la Fundación Paniamor y la Universidad de Costa Rica. La Fundación Paniamor llevó a cabo la encuesta y el autor principal de este artículo participó en el diseño, revisión de instrumentos, supervisión de la recolección y análisis en el marco del proyecto 723-B8-728, Vicerrectoría de Investigación, Universidad de Costa Rica. El segundo autor, participó en los análisis de resultados, redacción del artículo y revisión conceptual y de antecedentes.

${ }^{2}$ This article is a product of a collaborative work between the Paniamor Foundation and the University of Costa Rica. The Paniamor Foundation carried out the survey and the main author of this article participated in the design, review of instruments, supervision of the collection and analysis within the framework of project 723-B8-728 (University of Costa Rica). The second author participated in the analysis of results, writing of the article and conceptual review.

${ }^{3}$ Este artigo é produto de um trabalho colaborativo entre a Fundação Paniamor e a Universidade da Costa Rica. A Fundação Paniamor realizou a pesquisa e o autor principal deste artigo participou da concepção, revisão dos instrumentos, supervisão da coleta e análise no âmbito do projeto 723-B8-728, Universidade da Costa Rica. O segundo autor participou da análise dos resultados, redação do artigo e revisão conceitual.
} 
http://doi.org/10.15359/ree.26-1.1

http://www.una.ac.cr/educare

educare@una.ac.cr

\begin{abstract}
Resumen
Objetivo: El estudio investiga el efecto del tipo de mediación parental, las habilidades digitales, el género y el nivel socioeconómico en los usos de internet en niñas, niños y adolescentes (NNA) entre 9-17 años. Método: Para realizar esta investigación se tomaron los datos de la encuesta nacional Kids Online (PérezSánchez, 2019) aplicada a una muestra representativa de 1008 NNA costarricenses, 50,6\% mujeres y una edad promedio de $12,75(D T=2,55)$. Se siguió un muestreo aleatorio estratificado. Se realizaron modelos de ecuación estructural y análisis de moderación y mediación. Conclusiones: Los resultados muestran que los diferentes tipos de mediación contribuyen a promover o inhibir diferentes tipos de uso, resultando la mediación activa su principal promotor y el monitoreo su inhibidor, por encima de la mediación restrictiva. Las habilidades digitales tienen un efecto mediador parcial de la relación entre tipo de mediación y uso de internet, por lo que contribuyen a contrarrestar los efectos limitantes de la mediación por monitoreo o restrictiva sobre las oportunidades. Además, se encontró un efecto moderador del género y el nivel socioeconómico en la relación entre tipo de mediación y tipos de uso de la internet.
\end{abstract}

Palabras claves: Mediación parental; habilidades digitales; usos de internet; infancia; adolescencia.

\title{
Abstract
}

Objective: This study aimed to research the effects of parental mediation, digital skills, gender, and socioeconomic status on the Internet uses of children and adolescents between 9 and 17 years old. Method: The Kids Online survey (Pérez-Sánchez, 2019) was applied to a national representative sample of 1008 Costa Rican children and adolescents, 50.6\% women and an average age of 12.75 $(\mathrm{SD}=2.55)$. A stratified random sample was followed. Structural equation modeling and moderation, and mediation analysis were conducted. Conclusions: Results show that specific parental mediation types can contribute to promote or inhibit different Internet uses, resulting in active mediation as the main promoter and monitoring mediation as the main inhibitor, above restrictive mediation. Digital skills have a partial mediation effect in the association between types of mediation and Internet uses. This effect helps to counteract the limiting or restrictive effects of mediation on opportunities. Furthermore, a moderator effect of gender and socioeconomic status in the relationship between the type of mediation and Internet uses was found.

Keywords: Parental mediation; digital skills; Internet uses; childhood; adolescence.

\section{Resumo}

Objetivo: $O$ estudo investiga o efeito do tipo de mediação parental, das habilidades digitais, do gênero e do nível socioeconômico sobre o uso da Internet em crianças e adolescentes entre 9-17 anos. Método: para realizar esta investigação tomaram-se os dados da enquete costarriquenha Kids Online (Pérez-Sánchez, 2019) aplicada a uma amostra representativa de 1008 NNA costarriquenhos/ as, 50,6\% foram mulheres, e com a média de idade de 12.75 (DT $=2.55$ ). Utilizou-se uma amostra aleatória estratificada. Foram realizados modelos de equações estruturais e análises de moderação e mediação. Conclusão: os resultados mostram que os diferentes tipos de mediação contribuem a promover ou inibir distintos tipos de uso, tendo como resultado a mediação ativa como sua principal promotora e o monitoramento como seu inibidor, acima da mediação restritiva. As habilidades digitais têm um efeito mediador parcial na relação entre o tipo de mediação e o uso da internet. Esse efeito ajuda a neutralizar os efeitos limitantes ou restritivos da mediação sobre as oportunidades. Ademais, encontrou-se um efeito moderador do gênero e do nível socioeconômico na relação entre tipo de mediação e tipos de uso da Internet.

Palavras-chave: Mediação parental; habilidades digitais; usos da Internet; infância; adolescência. 


\section{Introducción}

El presente estudio se dirige a estudiar predictores relevantes de los usos de internet en niñas, niños y adolescentes (NNA). Específicamente, interesa estudiar la contribución del tipo de mediación parental y las habilidades digitales de las personas menores en los tipos de uso que hacen de internet, considerando igualmente el papel del género y las condiciones socioeconómicas.

NNA hacen uso de internet como parte de su vida cotidiana en atención a diferentes demandas sociales, personales o escolares y sobre la base de las tareas de desarrollo que tienen que afrontar en el día a día. De esta manera, internet es una herramienta cultural y tecnológica más para el desarrollo cognitivo y social, la formación educativa y la integración social (Livingstone et al., 2018).

Dentro de las tareas básicas de desarrollo asociadas al periodo del ciclo vital infantil y adolescente, están la apropiación reflexiva acerca de la identidad de sí, el desarrollo de relaciones cercanas, el afrontamiento con la propia sexualidad, así como el posicionamiento frente a la realidad social, el conocimiento acerca de su funcionamiento y las perspectivas ideológicas, sociales y políticas alrededor del devenir de la sociedad y el planeta (Livingstone y Third, 2017; Subrahmanyam y Smahel, 2011). Para cumplir con estas tareas básicas, se requiere el desarrollo de una serie de habilidades asociadas al desarrollo cognitivo general, las competencias comunicativas, el desarrollo de la autopresentación y de la autorrevelación (self-disclousre) (Subrahmanyam y Smahel, 2011; Valkenburg et al., 2011). Estos procesos sociocognitivos favorecen la adquisición de competencias básicas para manejarse e interactuar en internet y fuera de ella.

Se considera que los usos de internet deben ser visto en términos de oportunidades, entendiéndose por ellas, el espectro de posibilidades de uso al que pueden acceder las NNA. En este sentido las oportunidades no deben ser vistas únicamente desde la perspectiva de los beneficios. Por el contrario, beneficios y riesgos se entrelazan, además de estar marcados por la desigualdad social y la inequidad (Livingstone, Lemish et. al., 2017).

Según las investigaciones antecedentes realizadas tanto a nivel latinoamericano como en el contexto europeo, dentro de las oportunidades de usos de la internet se encuentran cuatro tipos básicos de uso (Cabello et al., 2018; Livingstone et al., 2018): i) El aprendizaje y educación, orientado a la búsqueda de información asociada a actividades escolares, intereses personales y conocimiento acerca de lo que acontece a su alrededor. ii) La comunicación, dirigida a mantener las interacciones con amistades o familiares que acontecen cara a cara, así como al desarrollo de nuevas relaciones acontecidas a nivel digital. iii) La participación ciudadana, asociada a formar parte en grupos comunitarios, vincularse a páginas informativas o directamente vinculadas a movimientos sociales o iniciativas ciudadanas. iv) Por último, el entretenimiento y la creatividad, dirigido a jugar en internet, al consumo o producción de material audiovisual, musical o literario o a la programación. 
http://doi.org/10.15359/ree.26-1.1

http://www.una.ac.cr/educare

educare@una.ac.cr

Se ha encontrado que los tipos y frecuencia de uso de la internet varían según la edad, el acceso a diferentes dispositivos, la experiencia de uso y el género, de modo que las personas que iniciaron antes su uso, los hombres, las personas con mayor acceso a dispositivos y las personas adolescentes son los que utilizan más esta tecnología (Livingstone et al., 2011; Mascheroni y Ólafsson, 2016).

En este artículo interesa estudiar la influencia del contexto familiar, particularmente el rol del parentaje, en el tipo de uso de la internet por parte de las personas menores de edad. A las formas de crianza asociadas a los usos de las tecnologías digitales se les ha llamado mediación parental (Valkenburg et al., 2013). Específicamente, interesa estudiar tres tipos de mediación (Livingstone, Ólafsson et al., 2017; Sonck et al., 2013): i) La mediación activa que se dirige a la guía y acompañamiento en el uso de la internet y sus aplicaciones asociadas, y supone padres y madres con las habilidades digitales suficientes para orientar tanto en la promoción del uso beneficioso como del riesgo. ii) La mediación restrictiva, se dirige a limitar el uso de determinadas páginas, aplicaciones o dispositivos, la limitación del tiempo de uso y restringir la comunicación. iii) La mediación por monitoreo, que se dirige a vigilar y auditar el tipo de páginas que visitan sus hijos o hijas, así como el tipo de personas con quienes se comunican.

El tipo de mediación restrictiva limita las oportunidades asociadas a los usos de la internet en los NNA, a la vez que cumple una función protectora que reduce los riesgos (Cabello-Hutt et al., 2018; Mascheroni y Ólafsson, 2016). Además, se ha encontrado que la mediación restrictiva reduce las habilidades digitales y con ello limita los usos de la internet (Rodríguez de Dios et al., 2018). De acuerdo con nuestro conocimiento, no existen antecedentes en la bibliografía sobre el impacto de la mediación de monitoreo en las habilidades digitales y los usos de la internet. No obstante, dadas ciertas similitudes conceptuales con la mediación restrictiva, es de esperar que la mediación de monitoreo se asocie negativamente con las habilidades digitales y el tipo de uso de la internet.

En cuanto a la mediación activa, Cabello-Hutt et al. (2018) encontraron, en Brasil, que los padres y madres, con alto nivel educativo y altas habilidades digitales son quienes recurren más a un tipo de mediación activa, lo cual se asocia a mayores habilidades digitales de los hijos o hijas y, de forma indirecta, a la promoción de las oportunidades de uso.

Por tanto, estos resultados demuestran la importancia de indagar, junto a la mediación parental, en las habilidades que tienen los NNA para hacer uso de las tecnologías digitales y sus efectos en los usos de las internet. Esta capacidad ha sido estudiada bajo el nombre de habilidades digitales. Las habilidades digitales se refieren al nivel de dominio sobre los usos de la internet; las posibilidades de distinguir entre tipos de información, ya sea confiable o falsa; los conocimientos que permiten resguardar la privacidad, mantener de forma adecuada las comunicaciones; así como conocimientos asociados al uso del browser, aplicaciones y transacciones en línea, junto con las posibilidades de creación de contenidos por parte de 
http://doi.org/10.15359/ree.26-1.1

los NNA. En el desarrollo de estas habilidades están implicadas competencias cognitivas y comunicativas espacializadas para el ecosistema digital, así como aprendizajes y conocimientos asociados al uso mismo de los programas o aplicaciones (van Dijk y van Deursen, 2014).

De forma consistente se ha encontrado una asociación positiva, tanto entre las habilidades digitales y las oportunidades, como con los riesgos en el uso de la internet (Cabello-Hutt et al., 2018; Livingstone, Ólafsson et al., 2017; Rodríguez de Dios et al., 2018). Asimismo, se ha encontrado que estas habilidades varían con la edad, pues se reportan mayores puntajes en adolescentes que en niñas y niños, como reportan Cabello et al. (2018) en población chilena.

Es esperable que todos los NNA no utilicen la internet de la misma manera, sino que la variedad en los repertorios de uso son influenciados por características sociodemográficas. Se considera relevante indagar en el efecto moderador -sobre la relación entre tipo de mediación parental y usos de la internet-de dos dimensiones tradicionalmente asociadas a la brecha digital: el género y la condición socioeconómica. Al respecto se han encontrado resultados disímiles. Tanto en Brasil como en el contexto europeo, las personas adolescentes hacen un mayor uso de los diferentes tipos de la internet (Cabello-Hutt et al., 2018; Mascheroni y Ólafsson, 2016). Otros estudios han encontrado diferencias en el uso de la internet para jugar o para la búsqueda de información. Sin embargo, los resultados varían dependiendo del país: en algunos casos los hombres, o en otros las mujeres, quienes emplean más en esas actividades (van Deursen y van Dijk, 2014); por su parte, Molina et al. (2017) encuentran mayores usos comunicativos y menores usos de entretenimiento por parte de las mujeres, en comparación con los hombres. Cabello et al. (2018) no encuentran diferencias entre hombres y mujeres a nivel comunicativo, un mayor uso dirigido a la participación ciudadana, por parte de las mujeres y mayor uso dirigido al entretenimiento por parte de los hombres.

En cuanto al nivel socioeconómico, se ha encontrado que jóvenes de niveles bajos utilizan más el celular, en comparación con niveles más altos, que tienen acceso a más dispositivos (Mascheroni y Ólafsson, 2016). Por otro lado, las NNA de niveles altos presentan mayores oportunidades en los usos de la internet (van Deursen y van Dijk, 2014).

Los resultados que se presentan a continuación prueban un modelo para estudiar el rol de los tipos de mediación parental y las habilidades digitales en los usos de la internet, así como aportar a la identificación de variables moderadoras o mediadoras de la relación entre tipo de mediación y oportunidades de uso, que a la fecha no han sido reportadas por la investigación antecedente consultada. Hasta ahora el grueso de la investigación antecedente no ha procedido de esta manera, por lo que los resultados que aquí se presentan son un aporte para la comprensión general del uso mediático. Además, hasta ahora el estudio no ha sido abordado en el contexto costarricense y centroamericano, por lo que sus resultados son significativos para entender el fenómeno desde la perspectiva de la comparación intercultural. 
http://doi.org/10.15359/ree.26-1.1

http://www.una.ac.cr/educare

educare@una.ac.cr

Las hipótesis del estudio son las siguientes:

H1: La mediación activa se asocia positivamente con los usos dirigidos al aprendizaje y la participación ciudadana.

H2: La mediación restrictiva y la de monitoreo se asocian negativamente con los usos dirigidos a la comunicación y el entretenimiento.

H3: Las habilidades digitales se asocian positivamente con todos los usos de la internet.

H4: Los usos orientados a la comunicación y el entretenimiento presentarán diferencias según el género.

H5: Las habilidades digitales co-varían negativamente con el tipo de mediación restrictiva y de monitoreo.

H6: Las NNA de condiciones socioeconómicas más altas harán mayor uso de la internet, independientemente del tipo de uso (estas hipótesis se representan en la Figura 1).

Figura 1: Determinantes de los usos de la Internet

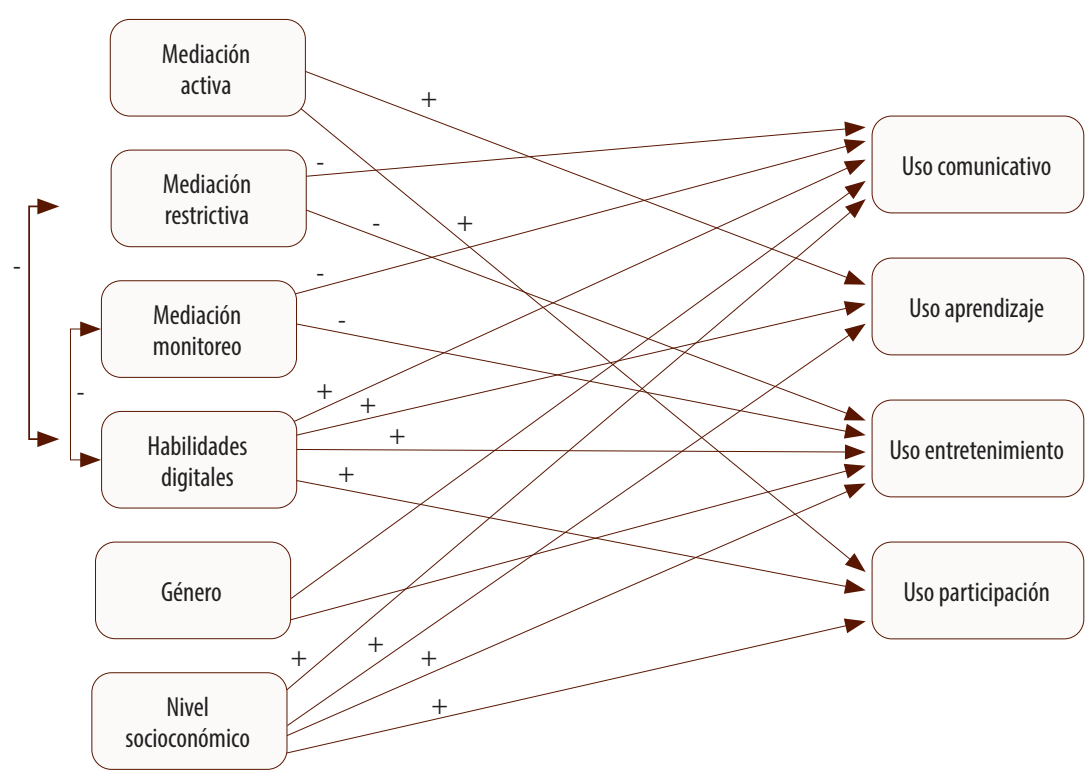

Nota: Elaboración propia. 
Una vez identificada la asociación de las variables sociodemográficas y del tipo de mediación sobre los usos, y considerando la investigación internacional antecedente, interesó indagar en el tipo de efecto que específicamente están ejerciendo, sea este moderador o mediador. Así, se consideraron las siguientes hipótesis:

H7: Las habilidades digitales van a tener un efecto mediador entre los tipos de mediación parental y los cuatro usos de la internet estudiados (ver Figura 2).

H8: El género y la condición socioeconómica tienen un efecto moderador sobre la asociación entre el tipo de mediación y los tipos de uso.

\section{Método}

Se realizó una encuesta nacional representativa de la población costarricense entre 9 y 17 años en el marco de la red Global Kids Online de Unicef.

\section{Participantes}

Se siguió un muestreo aleatorio, estratificado y polietápico que consideró como criterio de estratificación la zona geográfica, urbana o rural, controlando la distribución por edad y sexo de las unidades muestrales estudiadas. El tamaño muestral y el procedimiento de selección permite establecer estimaciones con un nivel de error máximo de 3\% y un nivel de confianza del 95\%.

Se aplicó un cuestionario a 1008 NNA entre 9 y 17 años (47,4\% de 9 a 12 años) que hubieran hecho uso de la internet en los últimos tres meses. El 50,6\% de las personas encuestadas son mujeres, con una edad promedio de 12,75 (DT =2,53). La encuesta se llevó a cabo en los hogares de las personas menores, con el previo consentimiento de la persona adulta cuidadora principal. El trabajo de campo se efectuo en el segundo semestre de 2018.

\section{Instrumentos}

Se recurrió al cuestionario de la encuesta Global Kids Online, tomando como referencia la versión aplicada en Chile (Cabello et al., 2019).

- Usos de la internet: Este instrumento se elaboró a partir del cuestionario utilizado en EU Kids Online (EUKO, 2014). Esta medida indaga en la frecuencia de diferentes usos de la internet. Considerando la investigación precedente se dividió en 4 tipos de uso: comunicativos, asociados a la comunicación con familia y amistades, usar una red social; información y aprendizaje orientados a tareas escolares; participación ciudadana, formar parte de actividades comunitarias o asociadas a protesta o reivindicaciones de diferente tipo; y entretenimiento, ligada con el juego o el consumo audiovisual. 
Se realizó un análisis factorial confirmatorio para probar la estructura factorial del constructo. El modelo encontró respaldo empírico de que los usos y las actividades asociadas están relacionados entre sí, obteniéndose resultados satisfactorios $\left(X^{2} 135\right.$, $p<.001$, CFI .97, TLI .96, SRMR .02, RMSEA .05 (CI [95\%] .04 - .06).

- Mediación parental: esta medida se construyó a partir del instrumento utilizado en (EUKO, 2014). Se refiere a los tipos de crianza asociados al uso de tecnologías digitales. Se estudiaron tres tipos: Mediación activa, se dirige al acompañamiento y guía, consta de 9 ítems (e.g. consejos sobre cómo usar la Internet de forma segura). Mediación restrictiva, el cual enfatiza en la prohibición de actividades en la internet, está formado por tres ítems (e.g. prohibición de ciertas páginas web). Monitoreo, el cual se dirige a vigilar el uso de la internet y consta de tres ítems (e.g. revisión de contactos o seguidores agregados al perfil de redes sociales). La medida de mediación restrictiva obtuvo un coeficiente alfa de .82, y las medidas de mediación activa y de monitoreo un alfa de .91.

- Habilidades digitales: la medida es una versión reducida del instrumento desarrollado por Helsper et al. (2015). Se refiere a las capacidades para el uso de la internet, las aplicaciones y dispositivos asociados (e.g. capacidad para reconocer qué información se debe evitar compartir en la internet). La medida unidimensional consta de 10 ítems y presenta un coeficiente alfa de .91.

- Junto con estas variables se consideró el sexo y el índice de condición socioeconómica: la sumatoria de un listado de bienes y servicios de que dispone la vivienda de la persona encuestada, junto con la pregunta de si el NNA posee su propia habitación, lo cual da como resultado una variable métrica.

\section{Procedimientos de recolección}

La encuesta fue revisada y adaptada a los usos de lenguaje castellano de Costa Rica. Posteriormente, se realizaron entrevistas cognitivas a 40 NNA de los grupos de edad en estudio, lo que permitió realizar correcciones para mejor la comprensión de los ítems. Seguidamente se realizó una prueba piloto a 100 participantes, lo que permitió hacer mejoras en el proceso de recolección.

Para la aplicación definitiva se realizó visita a hogares de las NNA. La encuesta fue aplicada por sujetos encuestadores previamente entrenados y se recurrió a un dispositivo móvil para recoger los datos.

\section{Procedimientos de análisis}

Los análisis incluyeron la realización de análisis descriptivos, correlación bivariada, determinación de consistencia interna para las medidas estandarizadas (habilidades digitales 
http://doi.org/10.15359/ree.26-1.1

y mediación parental), análisis factorial confirmatorio para probar la estructura interna de los usos de la internet. Una vez hechos estos análisis, se sometió a prueba un modelo de ecuación estructural para definir los predictores de los usos de la internet (tomando lo considerado por Hooper et al. (2008), seguido por la determinación del efecto moderador del sexo y el nivel socioeconómico, así como el efecto mediador de las habilidades digitales en la relación entre tipos de mediación parental y tipos de uso de la internet (según lo planteado por Hayes (2009) y Rucker et al. (2011).

\section{Resultados}

\section{Correlaciones bivariadas entre variables en estudio}

Como primer paso se presentan las correlaciones bivariadas entre los tipos de uso, los tipos de mediación parental y el puntaje en las habilidades digitales, así como entre estas y el nivel socioeconómico y el género (ver Tabla 1). El género se codificó como variable dummy (e.g., valor $0=$ mujer, $1=$ hombre) para posibilitar este tipo de análisis. A continuación, se reportan las asociaciones estadísticamente significativas.

Los usos de la internet presentan correlaciones positivas de moderadas a altas entre sí, que oscilan entre $r=.5$ y $r=.76$ (todos los valores $p<.001$ ). Los tipos de mediación presentan correlaciones moderadas positivas entre sí que oscilan entre $r=.41$ y $r=.64$ (valores $p<.001$ ).

Los tipos de mediación de monitoreo y restrictiva obtuvieron correlaciones negativas bajas o moderadas bajas con los usos orientados al entretenimiento, la comunicación y participación ciudadana (entre $r=.17$ a $r=.30$, valores $p<.001,<.01$ ) y se presenta además una correlación negativa baja entre la mediación restrictiva y los usos orientados al aprendizaje $(r=.08, p<.01)$. La mediación activa, por su lado, presenta asociaciones positivas bajas con todos los tipos de uso que oscilan entre $r=.10$ y $r=.31$ (valores $p<.001,<.01$ ).

Las habilidades digitales presentan correlaciones moderadas entre .38 y .52 (valores $p<.001$ ) con los tipos de uso, mientras que se presentan asociaciones negativas con la mediación restrictiva $(r=-.25, p<.001)$ y de monitoreo $(r=-.22, p<.001)$. Se encontró una correlación positiva baja entre la mediación activa y las habilidades digitales $(r=.16, p<.001)$.

El nivel socioeconómico se asocia de forma significativa con todas las variables en estudio, obteniéndose correlaciones positivas bajas o moderadas tanto con los tipos de uso $(r=.10$ a $r=.26$, valores $p<.001,<.01$ ) como con los tipos de mediación (entre $r=.09$ a $r=.25$, valores

$p<.001,<.01)$, resultando la mediación activa la correlación más alta. Las habilidades digitales también se asocian de forma positiva, pero baja con el nivel socioeconómico $(r=.14, p<.001)$. 
http://doi.org/10.15359/ree.26-1.1

http://www.una.ac.cr/educare

educare@una.ac.cr

Tabla 1: Matriz de correlación bivariada entre las variables en estudio

\begin{tabular}{|c|c|c|c|c|c|c|c|c|c|c|c|c|c|c|c|c|c|c|c|}
\hline & $M$ & SD & 1 & & 2 & & 3 & & & & 5 & & 6 & & 7 & & 8 & & 9 \\
\hline 1. Uso aprendizaje & 7.8 & 4.1 & - & & & & & & & & & & & & & & & & \\
\hline 2. Uso entretenimiento & 8 & 4.1 & .59 & $* * *$ & - & & & & & & & & & & & & & & \\
\hline 3. Uso comunicación & 7.9 & 5.4 & .52 & $* * *$ & .77 & $* * *$ & - & & & & & & & & & & & & \\
\hline 4. Uso participación & 3.4 & 3.5 & .49 & $* * *$ & .63 & $* * *$ & .65 & $* * *$ & - & & & & & & & & & & \\
\hline 5. Med. restrictiva & 6.2 & 4.4 & -.08 & ** & -.19 & $* * *$ & -.30 & $* * *$ & -.11 & $* * *$ & - & & & & & & & & \\
\hline 6. Med. monitoreo & 5.1 & 4.8 & -.04 & & -.17 & $* * *$ & -.27 & $* * *$ & -.18 & $* * *$ & .64 & $* * *$ & - & & & & & & \\
\hline 7. Med. activa & 3.5 & 3.7 & .31 & $* * *$ & .18 & $* * *$ & .11 & $* * *$ & .10 & $* *$ & .41 & $* * *$ & .43 & $* * *$ & - & & & & \\
\hline 8. Habilidades digitales & 7.5 & 2.4 & .38 & $* * *$ & .40 & $* * *$ & .52 & $* * *$ & .40 & $* * *$ & -.26 & $* * *$ & -.22 & *** & .16 & $* * *$ & - & & \\
\hline 9. Nivel socioeconómico & -- & -- & .25 & $* * *$ & .26 & $* * *$ & .17 & $* * *$ & .01 & $* *$ & .01 & $* *$ & .10 & $* *$ & .25 & $* * *$ & .14 & $* * *$ & - \\
\hline 10. Género & -- & -- & -.05 & & -.05 & & -.09 & $* *$ & -.10 & $* *$ & .02 & & -.02 & & -.07 & * & .03 & & .05 \\
\hline
\end{tabular}

${ }^{*} \mathrm{p}<.05,{ }^{* *} \mathrm{p}<.01,{ }^{* * *} \mathrm{p}<.001$. Género $=0=$ mujer, $1=$ hombre.

Nota: Elaboración propia.

En cuanto al género se encontró que se presenta una asociación significativa baja con los usos comunicativos $(r=-.09, p<.01)$ y participación ciudadana $(r=-.10, p<.01)$, específicamente, las mujeres usan más frecuentemente la internet para estos dos tipos de uso.

\section{La mediación parental, las habilidades digitales, el género y la condición socioeconómica como predictores de los tipos de uso de la internet}

El modelo propuesto para la predicción de los usos de la internet, considerando los tipos de mediación parental junto a las habilidades digitales, el género y la condición socioeconómica, como variables independientes, presenta un ajuste adecuado, $X^{2}(255)=475.8, p<.0001, \mathrm{CFI}=.96$, $\mathrm{TLI}=.95, \mathrm{RMSEA}=.04, \mathrm{IC}(90 \%)=.036-.047, \mathrm{SRMR}=.046, \mathrm{GFI}=.97, \mathrm{AGFI}=.96$.

Se encontró que los tipos de mediación covarían positivamente entre sí, de forma tal que altos puntajes en cualquiera de los tipos de mediación parental están asociados igualmente con puntajes altos en el resto de estrategias de mediación (relación mediación activa y restrictiva: $\beta=.51, I C=.43, .59, z=12.5, p=.0001$, mediación activa y monitoreo: $\beta=.48, I C=.41, .56$, $z=12.39, p=.0001$, mediación restrictiva y monitoreo: $\beta=.75, I C=.69, .80, z=27.24, p=.0001)$.

Además, se identificó una covariación negativa entre los tipos de mediación restrictiva y de monitoreo con las habilidades digitales, de modo que altos puntajes en estos tipos de mediación se vinculan con bajos puntajes en habilidades digitales (habilidades digitales y mediación restrictiva: $\beta=-.28, I C=-.37,-.18, z=-5.73, p=.0001$; habilidades digitales y monitoreo: $\beta=-.25, \mathrm{IC}=-.34,-.15, z=-5.28, p=.0001$. 
http://doi.org/10.15359/ree.26-1.1

Se encontró una asociación positiva entre la mediación parental activa y los usos comunicativos $(\beta=.12, \mathrm{IC}=.002, .32, z=1.99, \mathrm{p}=.05)$, de modo que altos puntajes de este tipo de mediación se relaciona con altos puntajes en el uso comunicativo. Caso contrario con lo que sucede con los otros tipos de mediación que presentaron asociaciones negativas, lo que implica que puntajes altos de mediación restrictiva $(\beta=-.23, \mathrm{IC}=-.056,-.09, \mathrm{z}=-2.72, \mathrm{p}=.007)$ y de monitoreo $(\beta=-.17, \mathrm{IC}=-.45,-.02 z=-2.28, \mathrm{p}=.0,02)$, se asocian con puntajes bajos en el uso orientado a la comunicación. Además, se determinó que las mujeres hacen mayor uso comunicativo que los hombres $(\beta=.-12, \mathrm{IC}=-.059,-.13, z=-3.05, \mathrm{p}=.002)$, así como las NNA de condición socioeconómica más alta hacen un mayor empleo vinculado a la comunicación $(\beta=.11, I C=.02, .11 z=2.68, p=.007)$.

En cuanto a los usos orientados al entretenimiento, se presentó una asociación positiva con la mediación activa $(\beta=.12, I C=.001, .31, z=1.97, p=.05)$, de forma tal que altos puntajes de este tipo de mediación se relaciona con altos puntajes en actividades de entretenimiento. Por el contrario, se encontró que altos puntajes de mediación de monitoreo se relacionan con puntajes bajos del uso orientado al entretenimiento $(\beta=-.25, \mathrm{IC}=-.54,-.12, z=2.68$, $\mathrm{p}=.007)$. Asimismo, se encontró que las mujeres hacen más frecuentemente actividades de entretenimiento que los hombres ( $\beta=-.10, I C=-.48,-.03, z=2.68, p=.007)$. De la misma manera, las NNA de niveles socioeconómicos más altos hacen un mayor uso asociado al entretenimiento $(\beta=15, I C=.03, .16, z=3.24, p=.001)$.

En lo referente al uso dirigido al aprendizaje y búsqueda de información, la mediación parental activa parece promover este tipo de uso $(\beta=.42$, IC $=.34, .70, z=5.68, p=.0001)$. En contraste, el predominio de mediación centrada en el monitoreo se asocia con la reducción del uso de la internet para el aprendizaje $(\beta=-.21, I C=-.49,-.05, z=2.42, p=.01)$.

Con respecto a la participación ciudadana, se pudo identificar que una mayor presencia de mediación activa se relaciona con un mayor empleo de la internet para la participación ciudadana ( $\beta=.23, \mathrm{IC}=.09, .49, z=2.82, \mathrm{p}=.005)$. De igual manera, se encontró que este tipo de uso se presenta más en mujeres que en hombres $(\beta=-.11$, IC $=-.57,-.009, z=2.68, p=.007)$.

Para los cuatro tipos de usos de internet estudiados, se encontró que las habilidades digitales se asocian positivamente con los usos de internet, de manera que mayores puntajes de habilidades digitales se relacionan con una mayor frecuencia en el uso de los diferentes tipos (uso comunicativo: activa: $\beta=.51, \mathrm{IC}=.56, .89, z=8.58, p=.0001$, entretenimiento: $\beta=.45$, $I C=.76, .43, z=7.602, p=.0001$, usos orientados al aprendizaje: $\beta=.36, I C=.30, .60, z=5.77$, $p=.0001$, usos dirigidos a la participación ciudadana: $\beta=.46, I C=.38, .78, z=5.69, p=.0001$ ) (ver Figura 2). 
http://doi.org/10.15359/ree.26-1.1

http://www.una.ac.cr/educare

educare@una.ac.cr

\section{Resumen de resultados}

En resumen, los resultados respaldan las hipótesis 1 a 5 , mientras que solo parcialmente la hipótesis 6. Como propuso la hipótesis 1, la mediación activa se asoció positivamente con los usos dirigidos al aprendizaje y la participación ciudadana. En cambio, la mediación restrictiva y la de monitoreo se asociaron negativamente con los usos dirigidos a la comunicación y entretenimiento $(\mathrm{H} 2)$. Por su parte, las habilidades sociales se asociaron positivamente con los cuatro usos de la internet $(\mathrm{H} 3)$. Se encontraron diferencias según género en los usos orientados a la comunicación y el entretenimiento, de manera que las mujeres utilizan más la internet para estos fines que los hombres (H4). En respaldo a la hipótesis 5, se encontró que las habilidades digitales covariaron negativamente con los tipos de mediación restrictiva y de monitoreo. Finalmente, las NNA de condiciones socioeconómicas más altas hacen un mayor uso de la internet para la comunicación y el entretenimiento, pero no así para el aprendizaje y la participación ciudadana (H6).

Figura 2: Predictores de los tipos de uso de Internet $\left(^{*}\right)$

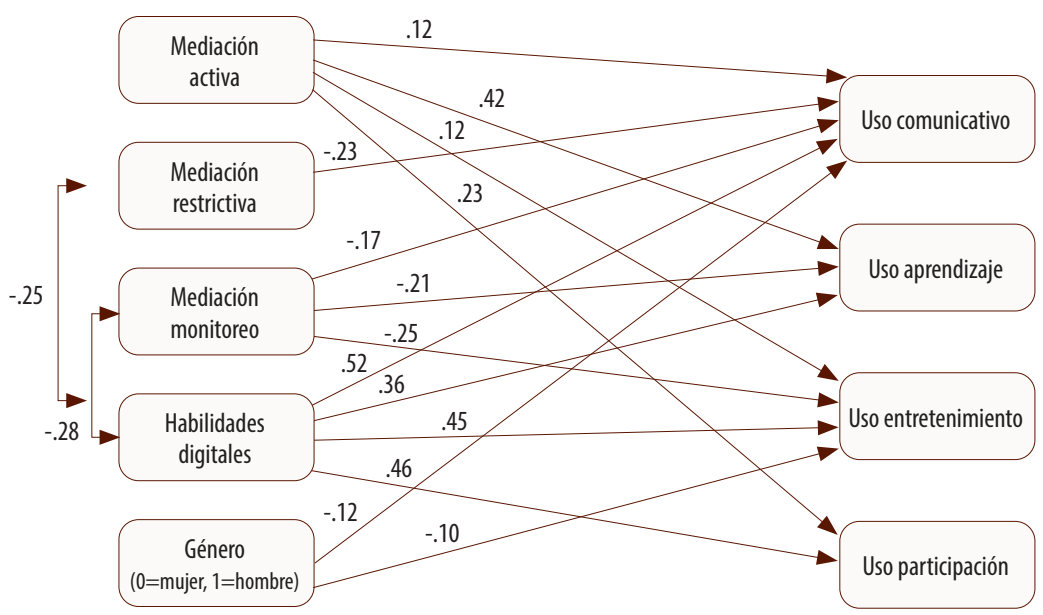

Nota: Elaboración propia.

\section{El efecto mediador de las habilidades digitales sobre la asociación entre tipo de mediación parental y usos de la internet}

Seguidamente se examinó la hipótesis 7, la cual establece que las habilidades digitales median la relación entre los tipos de mediación parental y los cuatro usos de la internet. Todos los resultados que a continuación se presentan dan cuenta de un tipo de mediación parcial en donde tanto el efecto directo y el efecto indirecto son significativos (Rucker et al., 2011; Zhao et al., 2010). 
http://doi.org/10.15359/ree.26-1.1 http://www.una.ac.cr/educare educare@una.ac.cr

Se encontró que las habilidades digitales tienen un efecto mediador sobre la asociación positiva entre mediación parental activa y el uso orientado al aprendizaje (Efecto total: Coef. .35, IC $[95 \%]=.26, .44, z=7.98, p<.001$. Efecto directo: Coef. .29, IC [95\%] = .21, .38, $z=6.98, p<.001$. Efecto indirecto: Coef. .06, IC [95\%] = .03, .10, $z=2.57, p<.001)$. Así, la mediación activa se asocia positivamente con las habilidades digitales (Coef. .11, IC [95\%] =.05, .16, $z=3.85, p<.001$ ), las habilidades con el uso orientado al aprendizaje (Coef. .58, IC [95\%] $=.46, .70, z=9.46, p<.001$ ) y la mediación activa con el uso orientado al aprendizaje (Coef. .29, IC [95\%] = .21, .38, z =6.98, $p<.001)$.

De igual manera, las habilidades digitales tienen un efecto mediador sobre la relación entre mediación parental activa y el uso dirigido a la participación ciudadana (Efecto total: Coef.: .14, IC [95\%] $=.06, .23, z=-3.34, p<.001$. Efecto directo: Coef.; .08, IC [95\%] = .003, .16, $z=2.06, p<.004$. Efecto indirecto: Coef.: .06, IC [95\%] =.003, .009, $z=3.42, p<.001)$. Así la mediación activa se asocia positivamente con las habilidades digitales (Coef. .10, IC [95\%] =.04, .16, $z=3.62, p<.001$ ), las habilidades con el uso orientado a la participación ciudadana (Coef. .60, IC [95\%] = .49, .71, $z=10.5, p<.001$ ) y esta relación favorece el efecto de la mediación activa sobre los usos vinculados a la participación ciudadana (Coef. .08, IC [95\%] = .003, .15, z= 2.05, $p<.04$ ).

Las habilidades digitales juegan como un factor catalizador del uso orientado al aprendizaje y a la participación ciudadana, ante el efecto promotor de la mediación activa.

Se encontró, además, que las habilidades digitales tienen un efecto mediador en las asociaciones negativas entre la mediación parental de monitoreo y los usos comunicativos, de entretenimiento y participación ciudadana (usos comunicativos, efecto total: Coef. -.34, IC [95\%] $=-.42,-.26, z=-7.96, p<.001$. Efecto directo: Coef. -.23, IC [95\%] = -.31, -.16, Z=6, $p<.001$. Efecto indirecto: Coef. -.11, IC [95\%] = -.16,-.06, $z=-4.94, p<.001$. Usos de entretenimiento, efecto total: Coef. -.19, IC [95\%] =. -26, -.13, z =9-6.1, $p<.001$. Efecto directo: Coef. -.14, IC [95\%] = -.2, -.08, $z=-4.46, p<.001$. Efecto indirecto: Coef. -.06, IC [95\%] $=-.08-.03, z=-4.52, p<.001$. Usos para la participación ciudadana, efecto total: Coef. -.13 IC [95\%] $=-.20,-.07, z=4.3, p<.001$. Efecto directo: Coef. -.07, IC [95\%] =. -13, -.02, $z=-2.49, p<.013$. Efecto indirecto: Coef. -.06, IC [95\%] $=$ $-.08,-.03, z=-4.62, p<.01)$.

De esta forma, se presentan asociaciones negativas entre el monitoreo y las habilidades digitales (Comunicación: Coef. -.11, IC [95\%] = -.015, -.07, z=-5.38, $p<.001$, entretenimiento: Coef. -.11 , IC [95\%] = -.14, -.07, z = -5.29, $p<.001$, participación ciudadana: Coef. -.11, IC [95\%] = -.15,-.07, $z=-5.41, p<.001)$. Por otro lado, una mayor puntuación en dichas habilidades aumenta tanto los usos comunicativos, los de entretenimiento y la participación ciudadana (Comunicación: Coef. 98, IC [95\%] = .83, 1.13, z= 12.55, $p<.001$, entretenimiento: Coef. .54, IC [95\%] = .42, .66, z = 8.69, $p<.001$, participación ciudadana: Coef. .54, IC [95\%] $=.42, .66, z=8.87, p<.001$ ). Por último, se presenta una asociación negativa entre la mediación de monitoreo y estos tres tipos de usos, 
http://doi.org/10.15359/ree.26-1.1

http://www.una.ac.cr/educare

educare@una.ac.cr

que precisamente van a estar mediadas por las habilidades digitales (Comunicación: Coef. -.23, IC [95\%] = -.30, -.16, $z=-6, p<.001$, entretenimiento: Coef. -.14, IC [95\%] = -.19, -.08, $z=-4.46$, $p<.001$, participación ciudadana: Coef. -.07, IC [95\%] $=-.13,-.02, z=-2.49, \mathrm{p}=.013)$.

Se pudo identificar que las habilidades digitales también tienen un efecto mediador en las asociaciones negativas entre la mediación parental restrictiva y los usos comunicativos, de entretenimiento y de participación ciudadana (Usos comunicativos, efecto total: Coef. -.42, IC $[95 \%]=-.51,-.32, z=-9.05, p<.001$. Efecto directo: Coef. -.28, IC [95\%] $=-.37,-.20, z=$ 6.73, $p<.001$. Efecto indirecto: Coef. -.13, IC [95\%] $=-.18,-.09, z=-5.614, p<.001$. Usos de entretenimiento, efecto total: Coef. -.21, IC [95\%] =. -28, -.14, $z=-5.96, p<.001$. Efecto directo: Coef. -.13, IC [95\%] = -.2, -.06, $z=-3.94, p<.001$. Efecto indirecto: Coef. -.09, IC [95\%] = -.11 -.05, $z=-5.29, p<.001$. Usos para la participación ciudadana, efecto total: Coef. -.18, IC [95\%]= -.25, $-.12, z=5.41, p<.001$. Efecto directo: Coef. -.10, IC [95\%] = -.17, -.04, $z=-3.21, p<.001$. Efecto indirecto: Coef. -.08, IC [95\%] $=-.10,-.05, z=-5.34, p<.001)$.

De esta manera, se presentaron asociaciones negativas entre la mediación restrictiva y las habilidades digitales (Comunicación: Coef. -.14, IC [95\%] $=-.018,-.09, z=-6.26, p<.001$, entretenimiento: Coef. -.14, IC [95\%] = -.18, -.10, $z=-6.58, p<.001$, participación ciudadana: Coef. -.14, IC [95\%] = -.19, -.10, $z=-5.41, p<.001)$. Mientras que mayores puntuaciones en estas habilidades están relacionadas con mayores usos comunicativos, los de entretenimiento y los de participación ciudadana (Comunicación: Coef. 97, IC [95\%] $=.82,1.12, z=12.73, p<.001$, entretenimiento: Coef. .56, IC [95\%] =.44, .68, $z=9.14, p<.001$, participación ciudadana: Coef. .53 , IC $[95 \%]=.42, .65, z=9.15, p<.001)$. Finalmente, se presenta una asociación negativa entre la mediación restrictiva y estos tres tipos de usos, que precisamente van a estar mediadas por las habilidades digitales (Comunicación: Coef. -.28, IC [95\%] $=-.037,-.20, z=-6.73, p<.001$, entretenimiento: Coef. -.13, IC [95\%] = -.20, -.06, $z=-3.84, p<.001$, participación ciudadana: Coef. -.07, IC [95\%] = -.13, -.02, $z=-2.49, p<.001)$.

\section{Resumen de resultados}

Estos resultados sugieren que el efecto inhibidor de la mediación de monitoreo y la mediación restrictiva sobre los usos comunicativos, de entretenimiento y de participación ciudadana se ven reducidos por las habilidades digitales. Por tanto, los resultados anteriores respaldan la hipótesis 7.

\section{El efecto moderador del sexo y la condición socioeconómica en la asociación entre mediación parental y usos de la internet}

Finalmente se sometió a prueba la hipótesis 8 , la cual sugiere que el género y la condición socioeconómica moderan la relación entre los tipos de mediación y los tipos de uso. El género tiene un efecto moderador en la asociación negativa entre la mediación parental restrictiva y los 
http://doi.org/10.15359/ree.26-1.1

usos orientados al aprendizaje (Coef. $=.13, \mathrm{IC}[95 \%]=.01, .24, z=2.13, p=.03$ ), resulta que son las mujeres las que hacen mayor uso de la internet para el aprendizaje en el contexto de un tipo de mediación restrictiva (Simple slope analysis, coef. $=-.14$, IC $[95 \%]=-.22,-.06, z=-3.32, p<.001$ ).

El estatus socioeconómico tiene un efecto moderador sobre la asociación positiva entre la mediación activa y los usos orientados a la comunicación (Coef. $=-.04, \mathrm{IC}[95 \%]=-.08,-.005, z=-2.26$, $p=.02$ ), el entretenimiento (Coef. $=-.04$, IC $[95 \%]=-.07,-.002, z=-3.14, p=.002$ ) y el aprendizaje (Coef.= -.04, IC [95\%] = -.06, -.01, z =-2.70, $p=.007$ ), de modo que en los niveles socioeconómicos más bajos es donde más se ejerce este tipo de mediación en relación con los usos orientados a la comunicación (Simple slope analysis, coef. $=.21$, IC $[95 \%]=.09, .34, z=3.30, p<.001$ ) y el entretenimiento (Simple slope analysis, coef. $=.24$, IC $[95 \%]=.15, .33, z=5.13, p<.001$ ).

Similar sucede con el efecto moderador de esta variable sobre la mediación parental de monitoreo y los usos comunicativos, de participación ciudadana (Coef. $=-.02$, IC $[95 \%]=-.05$, $-.005, z=-2.45, \mathrm{p}=.014$ ) y aprendizaje (Coef. $=-.04$, IC $[95 \%]=-.04,-.005, z=-2.42, \mathrm{p}=.002$ ). De igual manera, las NNA de condición socioeconómica más alta es donde menos se presenta este tipo de mediación parental asociado a estos usos de la internet (Simple slope analysis, aprendizaje, coef. $=-.12$, IC [95\%] $=-.19,-.04, z=-3.01, p=.003$; participación ciudadana, coef. $=-.20$, IC $[95 \%]=-.26,-.13, z=-5.70, p<.001)$.

Por tanto, los resultados respaldan la hipótesis 8.

\section{Discusión}

El presente estudio investigó el efecto de tres tipos de mediación parental en cuatro tipos de uso de la internet entre NNA costarricenses. Además, se examinó el rol mediador de las habilidades sociales de NNA en la relación entre tipos de mediación y usos de la internet. Finalmente, se estudió el efecto moderador del género y el nivel socioeconómico en la relación entre mediación y usos.

Los resultados dan respaldo empírico a las hipótesis $\mathrm{H1}, \mathrm{H} 2, \mathrm{H} 3, \mathrm{H} 4$ y $\mathrm{H} 5, \mathrm{H} 7, \mathrm{H} 8$ y respaldo parcial a la H6. En todos los casos se encontraron hallazgos emergentes relacionados con las hipótesis, los cuales son particularmente relevantes, en el contexto de la investigación internacional y las repercusiones locales de estos resultados.

La mediación parental activa, dirigida a la guía y acompañamiento en los usos de la internet, parece tener un efecto promotor de los tipos de uso, independientemente de la actividad. La mediación de monitoreo, aquella asociada a vigilar las actividades en internet parece que tiene un efecto inhibidor general de las oportunidades de uso, independientemente del tipo. La mediación restrictiva, orientada a prohibir y limitar el uso, parece que solo tiene un efecto inhibidor en el uso orientado a la comunicación, al estudiar la muestra total. 
http://doi.org/10.15359/ree.26-1.1

http://www.una.ac.cr/educare

educare@una.ac.cr

Como se hipotetizó y se ha encontrado en la bibliografía latinoamerica e internacional, en general (Cabello-Hutt et al., 2018; Livingstone, Ólafsson et al., 2017; Rodríguez de Dios et al., 2018), un mayor dominio de las habilidades digitales predice también todos los diferentes tipos de uso, de modo que un mayor dominio de la internet, el browser, las aplicaciones asociadas y las medidas de protección y mantenimiento de la privacidad favorecen un mayor empleo de las diferentes oportunidades de uso en estudio.

Se encontró, de igual manera, que tanto la mediación restrictiva como la de monitoreo tienden a inhibir tanto las habilidades digitales como los usos de la internet. Como encontraron Livingstone, Ólafsson et al. (2017), estos tipos de mediación tienen un efecto protector, al reducir los riesgos por limitar el uso, pero también restringen los usos beneficiosos y las capacidades básicas para el uso de la internet. Esta actividad atribuida a las madres y padres impide el empleo autónomo de la internet, por lo que empobrece las oportunidades asociadas a esta tecnología para la integración social, el aprendizaje y la introducción a la actividad económica y ciudadana.

Es necesario que futuras investigaciones estudien con mayor profundidad por qué tanto la mediación restrictiva y la de monitoreo inhiben las habilidades digitales, pero en cuanto a los usos de la internet, la mediación de monitoreo limita un mayor repertorio de usos, en comparación con la medición restrictiva.

Resulta destacable que no se encontró que la mediación activa facilitara las habilidades digitales. Al respecto es necesario estudiar para futuras investigaciones las habilidades digitales de las madres y padres, para comprender con mayor detalle las destrezas que pueden estar sustentado la mediación activa. Además, es importante estudiar otras fuentes de aprendizaje de habilidades digitales entre los NNA, por ejemplo, el aprendizaje por sí con base en sus experiencias en el uso de tecnologías, el aprendizaje mediado entre pares y aquel que proviene de la educación formal.

Se encontró que, salvo por el uso orientado a la búsqueda de información y el aprendizaje, las mujeres usan más la internet que los hombres para la comunicación, el entretenimiento y la participación ciudadana. Molina et al. (2017) encuentran en España, de igual manera, que las mujeres utilizan en mayor medida la internet para la comunicación, mientras que Cabello et al. (2018), para el contexto chileno, señalan que hay un mayor uso para la participación ciudadana por parte de mujeres. Como ya se ha indicado, no parecen encontrarse diferencias claras entre hombres y mujeres que se mantengan interculturalmente, por lo que resulta relevante atender a las particularidades culturales para tratarlo de entender. En el caso costarricense, estas diferencias podrían comprenderse en el marco del significado que tiene la internet y su uso a través del teléfono celular, dirigido a la sociabilidad, aspecto que puede ser entendido como un elemento diferenciador dentro de la construcción cultural de los roles de género. 
http://doi.org/10.15359/ree.26-1.1

Se encontró respaldo parcial sobre la asociación entre condición socioeconómica y tipo de uso, logrando identificarse que los usos comunicativos y de entretenimiento se presentan más en los niveles socioeconómicos más altos. Los usos ligados al aprendizaje y participación ciudadana parecen no depender de esta variable contextual. Comunicación y entretenimiento resultan ser los principales usos a los que recurren NNA, el hecho de que exista una brecha digital, asociada al acceso y tenencia, determinada por la condición socioeconómica en el país (PérezSánchez, 2019), puede explicar que se dé mayor uso en estos niveles socio-económicos. Ahora bien, el hecho de que no haya diferencias en lo referente al empleo orientado al aprendizaje y la participación podría indicar que esta tecnología es una fuente de reducción de dicha brecha en lo que se refiere a estas actividades. Se requiere mayor investigación que permita profundizar en estos resultados.

Se logró identificar un efecto mediador de las habilidades digitales sobre la relación entre la mediación parental y los diferentes usos. En el caso de la mediación activa, las habilidades digitales favorecen al establecimiento de una asociación positiva con los usos dirigidos a la participación ciudadana y el aprendizaje. Las habilidades digitales favorecen los usos a pesar del efecto inhibidor de la mediación de monitoreo y restrictiva, o bien lo incrementan, si las habilidades digitales de la NNA son bajas. Específicamente, esto aplica para los usos comunicativos, de entretenimiento y participación ciudadana. Este resultado, que parece no haber sido estudiado, puede indicar que el desarrollo de las habilidades digitales se lleva a cabo con cierta independencia de la crianza parental y que incluso el efecto facilitador de la mediación activa se ejerce paralelamente al desarrollo de estas habilidades. ¿Cuáles son las fuentes del desarrollo de las habilidades digitales? Y, ¿cuál es específicamente el efecto de la crianza parental en su facilitación o inhibición? Estas interrogantes requieren de futuras investigaciones.

Estas preguntas son importantes porque estos hallazgos sugieren que NNA con altas habilidades aprovechan la diversidad de ofertas de uso de la internet con cierta independencia de la influencia de la crianza parental en el ámbito tecnológico. Aunque dicha interpretación debe someterse a una prueba empírica, se debe destacar que los resultados de este estudio indican que los mayores beneficios para el uso de la internet entre NNA provienen de una combinación entre una mediación parental activa y que NNA posean habilidades digitales.

Se identificó un efecto moderador del género sobre la asociación negativa entre mediación restrictiva y el uso orientado al aprendizaje, donde los hombres son los que se ven más limitados en este tipo de uso por este tipo de crianza digital. Este resultado requiere de mayor investigación, ya que parece estarse limitando las oportunidades educativas recurriendo a la internet, en el caso de los hombres.

De igual manera, se determinó un efecto moderador del nivel socioeconómico sobre la relación positiva entre la mediación activa y el uso orientado al aprendizaje resultando que es en los niveles socioeconómicos más bajos donde se presenta más esta asociación. Este resultado es 
http://doi.org/10.15359/ree.26-1.1

http://www.una.ac.cr/educare

educare@una.ac.cr

relevante de estudiar con mayor detenimiento, ya que podría indicar que el efecto beneficioso de la mediación activa se ve disminuido debido a condiciones propias de la desigualdad socioeconómica, que podrían estar asociadas con las habilidades digitales o el nivel educativo de las madres o los padres, de los niveles socioeconómicos más bajos. Además, se encontró que, en las NNA de condición socioeconómica más alta, es donde menos se presenta la asociación negativa entre mediación de monitoreo y los usos de la internet orientados a la participación ciudadana, lo que podría indicar que el efecto de la acción inhibidora de este tipo de mediación es menor en los niveles más altos en este tipo de uso, posiblemente debido a condicionantes señalados para el resultado anterior, en este caso debido a mayores habilidades digitales o educación en esta condición socioeconómica.

Para próximos estudios es necesario continuar indagando, de forma más detallada en las diferencias por género y nivel socioeconómico. Así mismo se requiere realizar otros análisis que permitan indagar en las diferencias por edad. Relevante también es determinar el efecto de las habilidades digitales y los tipos de uso de los padres y madres, en el modelo acá estudiado.

En conclusión, internet es una herramienta social y tecnológica importante para el abordaje de tareas básicas de los ciclos vitales de NNA. Este estudio demuestra que en un contexto en donde los padres y madres practican una mediación activa y en el que NNA poseen habilidades digitales, es más probable maximizar los beneficios de los usos de la internet y proteger a la niñez y adolescencia de sus riesgos.

\section{Declaración de Material complementario}

Este artículo tiene disponible, como material complementario:

-La versión preprint del artículo en https://doi.org/10.5281/zenodo.4906578

\section{Referencias}

Cabello, P., Claro, M., Lazcano-Peña, D. y Antezana, L. (2018). La inclusión digital de niños y adolescentes chilenos desde la perspectiva de usos y habilidades. En E. Jiménez, M. Garmendia y M. Á. Casado (Coords.), Entre selfies y whatsapps. Oportunidades y riesgos para la infancia y la adolescencia conectada (pp. 259-278). Gedisa.

Cabello, P., Claro, M., Lazcano, D., Cabello-Hutt, T., Antezana, L. y Ochoa, J. M. (2019). Chilean children's internet use and online activities: A brief report. Global Kids Online http:// globalkidsonline.net/wp-content/uploads/2017/07/Chile-findings-report-FINAL.pdf 
http://doi.org/10.15359/ree.26-1.1

Cabello-Hutt, T., Cabello, P. y Claro, M. (2018). Online opportunities and risks for children and adolescents: The role of digital skills, age, gender and parental mediation in Brazil. New Media \& Society, 20(7), 2411-2431. https://doi.org/10.1177/1461444817724168

EU Kids Online. (2014). Research toolkit. LSE. http://globalkidsonline.net/tools/survey/

Hayes, A. F. (2009). Beyond Baron and Kenny: Statistical mediation analysis in the new millennium. Communication Monographs, 76(4), 408-420.https://doi.org/10.1080/03637750903310360

Helsper, E. J., van Deursen, A. J. A. M. y Eynon, R. (2015) From digital skills to tangible outcomes: Full questionnaire. https://www.Ise.ac.uk/media-and-communications/assets/documents/ research/projects/disto/From-Digital-Skills-to-Tangible-Outcomes-Full-Questionnaire..pdf

Hooper, D., Coughlan, J. y Mullen, M. R. (2008). Structural equation modelling: Guidelines for determining model fit. Electronic Journal of Business Research Methods, 6(1), 53-60. https:// academic-publishing.org/index.php/ejbrm/article/view/1224/1187

Livingstone, S., Haddon, L., Görzig, A. y Ólafsson, K. (2011). Risks and safety on the internet: The perspective of european children. Full finding and policy implications from the EU Kids Online survey of 9-16 year olds and their parents in 25 countries. EU Kids Online. http://eprints.Ise. ac.uk/33731/

Livingstone, S., Lemish, D., Lim, S. S., Bulger, M., Cabello, P., Claro, M., Cabello-Hutt, T., Khalil, J., Kumpulainen, K., Nayar, U. S., Nayar, P., Park, J., Tan, M. M. Prinsloo, J. y Wei, B. (2017). Global perspectives on children's digital opportunities: An emerging research and policy agenda. Pediatrics, 140(Supplement 2), S137-S141. https://doi.org/10.1542/peds.2016-1758S

Livingstone, S., Ólafsson, K., Helsper, E. J., Lupiáñez-Villanueva, F., Veltri, G. A. y Folkvord, F. (2017). Maximizing opportunities and minimizing risks for children online: The role of digital skills in emerging strategies of parental mediation. Journal of Communication, 67(1), 82-105. http://dx.doi.org/10.1111/jcom.12277

Livingstone, S., Mascheroni, G. y Staksrud, E. (2018). European research on children's internet use: Assessing the past and anticipating the future. New Media \& Society, 20(3), 1103-1122. https://doi.org/10.1177/1461444816685930

Livingstone, S.yThird, A. (2017). Children and young people's rights in the digital age: An emerging agenda. New Media \& Society, 19(5), 657-670. https://doi.org/10.1177/1461444816686318

Mascheroni, G. y Ólafsson, K. (2016). The mobile internet: Access, use, opportunities and divides among european children. New Media \& Society, 18(8), 1657-1679. https://doi. org/10.1177/1461444814567986

Molina, J. A., Campaña, J. C. y Ortega, R. (2017). Children's interaction with the internet: Time dedicated to communications and games, Applied Economics Letters, 24(6), 359-364, https://doi.org/10.1080/13504851.2016.1192270 
http://doi.org/10.15359/ree.26-1.1

http://www.una.ac.cr/educare

educare@una.ac.cr

Pérez-Sánchez, R. (2019). Informe primera encuesta Kids Online Costa Rica. Niñas, niños y adolescentes en la internet. Fundación Paniamor; IIP, UCR. http://www.iip.ucr.ac.cr/es/ publicaciones/publicacion-de-investigador/informe-primera-encuesta-kids-onlinecosta-rica-abril

Rodríguez de Dios, I., van Oosten, J. e Igartua, J. J. (2018). A study of the relationship between parental mediation and adolescents' digital skills, online risks and online opportunities. Computers in Human Behavior, 82, 186-198. https://doi.org/10.1016/j.chb.2018.01.012

Rucker, D. D., Preacher, K. J., Tormala, Z. L. y Petty, R. E. (2011). Mediation analysis in social psychology: Current practices and new rsecommendations. Social and Personality Psychology Compass, 5(6), 359-371. https://doi.org/10.1111/j.1751-9004.2011.00355.x

Subrahmanyam, K. y Smahel, D. (2011). Digital youth. The role of media in development. Springer. https://doi.org/10.1007/978-1-4419-6278-2

Sonck, N., Nikken, P. y de Haan, J. (2013). Determinants of internet mediation: A comparison of the reports by dutch parents and children. Journal of Children and Media, 7(1), 96-113. https://doi.org/10.1080/17482798.2012.739806

Valkenburg, P. M., Piotrowski, J.T., Hermanns, J.y de Leeuw, R. (2013). Development and validation of the perceived parental media mediation scale: A self-determination perspective. Human Communication Research, 39(4), 445-469. https://doi.org/10.1111/hcre.12010

Valkenburg, P. M., Sumter, S. R. y Peter, J. (2011). Gender differences in online and offline self-disclosure in pre-adolescence and adolescence. British Journal of Developmental Psychology, 29(2), 253-269. https://doi.org/10.1348/2044-835X.002001

Van Deursen, A. J. A. M. y van Dijk, J. A. G. M. (2014). The digital divide shifts to differences in usage. New Media \& Society, 16(3), 507-526. https://doi.org/10.1177/1461444813487959

van Dijk, J. A. G. M. y van Deursen, A. J. A. M. (2014). Digital skills: Unlocking the information society. Palgrave-Macmillan. https://doi.org/10.1057/9781137437037

Zhao, X., Lynch, J. G., Jr., y Chen, Q. (2010). Reconsidering Baron and Kenny: Myths and truths about mediation analysis. Journal of Consumer Research, 37(2), 197-206. https://doi. org/10.1086/651257 